\title{
(DCNN) Deep Convolution Neural Network Classifier and (EW-CSA) Earth Worm-Crow Search Algorithm for Lane Detection
}

\author{
Suvarna Shirke, R.Udayakumar
}

\begin{abstract}
Every year in India, most of the car accidents are occurs and affects on number of lives. Most of the road accidents are occurs due to driver's inattention and fatigue. Drivers require to focus on different circumstances, together with vehicle speed and path, the separation between vehicles, passing vehicles, and potential risky or uncommon events ahead. Also the accident occurs due to the who bring into play cell phones at the same time as driving, drink and drive, etc. Due to this, most of the companies of automobiles tries to make available best Advanced Driver Assistance System (ADAS) to the customer to avoid the accidents. The lane detection approach is one of the method provided by automobile companies in ADAS, in which the vehicle must follows the lane. Therefore, there is less chance to get an accident. The information obtained from the lane is used to alert the driver. Therefore most of the researchers are attracted towards this field. But, due to the varying road circumstances, it is very difficult to detect the lane. The computer apparition and machine learning approaches are presents in most of the articles. In this article, we presents the deep learning scheme for identification of lane. There are two phases are presents in this work. In a first phase the image transformation is done and in second phase lane detection is occurred. At first, the proposed model gets the numerous lane pictures and changes the picture into its relating Bird's eye view picture by using Inverse perspective mapping transformation. The Deep Convolutional Neural Network (DCNN) classifier to identify the lane from the bird's eye view image. The Earth Worm-Crow Search Algorithm (EW-CSA) is designed to help DCNN with the optimal weights. The DCNN classifier gets trained with the view picture from the bird's eye image and the optimal weights are selected through newly developed EW-CSA algorithm. All these algorithms are performed in MATLAB. The simulation results shows that the exact detection of lane of road. Also, the accuracy, sensitivity, and specificity are calculated and its values are $0.99512,0.9925$, and 0.995 respectively.
\end{abstract}

Keywords : Driver Help System (DAS), Deep Convolution Neural Network(DCNN), Earth Worm-Crow Search Algorithm $(\boldsymbol{E W}-\mathrm{CSA})$, etc.

\section{INTRODUCTION}

Now a days, due to the lot of traffic many of road accidents are occurs in India. There are many reasons to occurs these accidents some of them are due to careless of driver. some drivers sleeps while driving; Therefore in many

Revised Manuscript Received on November 05, 2019.

Suvarna Shirke, Research Scholar, Computer Science and Engineering Department, Bharath Institute of Higher Education and Research, Chennai, India. Worked as Assistant Professor at Atharva College of Engineering.

Dr.R.Udayakumar, Professor and Supervisor, Department of Information Technology, Bharath Institute of Higher Education and Research, Chennai, India of the real time applications the warning of lane departure is given to the driver to assist them. This system is called Lane Departure Warning (LDW) system. In this LDW method the warning is to be given to the driver if the vehicle goes in outside the lane and to avoid this incorrect path of lane, this LDW system takes automatic action.

The security of driving is depends on the driver and this LDW system. This LDW method uses the lane marking for keeping the position of vehicle constant and in between the two lanes[5]. Therefore the marking presents on the road for lane plays an important role for this LDW system. But these lane marking are not much clear due to the different conditions of environment; conditions due to light the detection of lane is quite difficult also due to the poor quality of material used for construction of road, the patches are occurs on the marking for lane. Also due to the trees presents outsides the road and neighboring vehicles the shadows falls on the lane marking therefore in this condition the LDW system cannot recognize the lane. Also the marking of tyres are presents on the lane strips, these marking are also responsible for incorrect lane identification[33].

The Indian governments takes more actions to avoid road accidents, the use of seat belt, air bags and speed limit is mandatory on highway. Also, in most of the vehicles an intelligent on-board system is provided to assists the driver. This on-board system plays an important role to avoid the accidents. In most of the article, these intelligent on board system is called as Advance Driver Assistant system (ADAS). In the complicated driving conditions this ADAS helps drivers for driving by proving proper signals. In most of the application, the ADAS system utilize the camera to capture or track the lanes presents on the road. If the vehicle driver drives wrongly or goes in intentionally out of lane then the ADAS system gives him the notification and take the correct action to avoid the incorrect lane. In this way the vehicle automatically comes in the correct lane. [6]. But the lane marking sometimes completely disappears due to the heavy traffic conditions as discussed above.[22].

Many technologies have emerged in past decade for assisting the driver, and the notable research is the development of the Lane Departure advice (LDW) structure. A LDW system alerts a driver, in case of change in lane or deviation from the actual lane. The LDW model assists the driver during most cases, and mostly beneficial for the narrow road or arterial roads and freeways. 


\section{(DCNN) Deep Convolution Neural Network Classifier and (EW-CSA) Earth Worm-Crow Search Algorithm for Lane Detection}

Besides, the LDW model prevents unnecessary alarm by setting a speed constraint. Due to the advantages, the LDW can assist the lousy drivers and avoid accidents and crashes in the road. The advancement in the technology has lead to the development of the visualization based lane finding for the LDW system [11]. A lane recognition methods frequently believe the color or edge information of the image samples for detecting the lane. In most cases, the road lanes have contrasting color labels, and hence, detecting the road lanes through the color cue can be considered as the added advantage [12].

In the practical scenario, the environmental conditions, like changing illumination, backlight, night time, and rainy conditions, have a major influence on the color feature. Hence, considering only the color cue for the lane detection is limited for the small roads and lanes [13]. Lane recognition is single of the solution fundamentals of adaptive cruise control (ACC) [14] and it aids in developing a warning system. The identification of lane calculation information of color and edge. For the identification of lane is the ACC is one of the key element. The sample images for the detection model may be affected by the noise as the images are subjected to variation due to the different environmental scenarios. Some practical scenarios include change in light, and uneven paintings etc, may affect the detection task [15]. Thus, to improve the performance of the algorithms some conditions like as invariable path size, reliable path surface, invariable track painting size and smooth path are considered [4].

The fundamental point of this paper is to plan and build up a propelled Driver-help framework for the solid recognition of street paths. The numerous path pictures are acquired and at the initial step, the pictures are given as the contribution of the driver help system[33]. At that point, the information pictures are changed into its relating view picture from the bird's eye utilizing the Inverse viewpoint mapping change process, which includes the utilization of cameras on the vehicle to join the perspectives from the cameras. This change method gives the driver an unmistakable picture of the territory behind the vehicle. The superior view picture is the contribution to the Deep Convolutional Neural Network $(\mathrm{DCNN})$ classifier.

The DCNN [23] is used in the process of lane detection, in which the weight values are estimated with the newly developed Earth Worm- Crow Search Method (EW-CSA). A proposed EW-CSA is developed by the integration of the algorithms, such as Earth Worm Optimization (EWA) [24] and Crow Search Algorithm (CSA) [25]. The proposed EW-CSA algorithm inherits the advantages of both the algorithms and it tunes the weights of the DCNN networks optimally for the successful lane finding. Figure 1 shows the example of identification of land of road.

The rest part of this article is in following way. In section 2 the literature work is present which helps to previous working methodologies and ideas to build this article. In section 3 different challenges are presents while designing this work. In fragment 4 the stream of the planned system and different method used is present. The block illustration of planned scheme is presents in section 5. Section 6 shows the investigational outcome of the planned system. At last, the conclusion is presents in segment 7 .

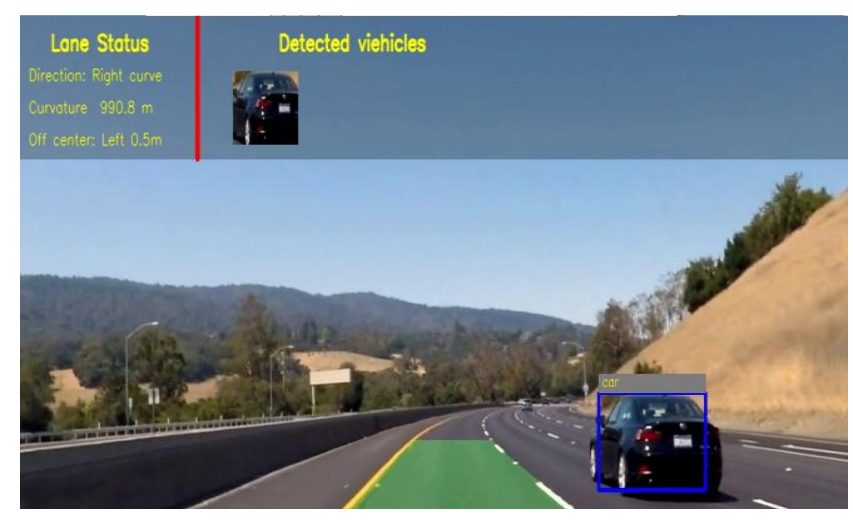

Fig. 1. Example Of Identification Of Lane Of Road [34].

\section{LITERATURE WORK}

A portion of the writing works created in the ongoing years for the lane discovery are clarified below:

Umar Ozgunalp et al. [1] proposed a lane detection algorithm for detecting various lanes from the sample image. The scheme allows detection of the lane roads even though the traffic was considered to be dense and high. The authors have manually setup the vehicle using air-suction pads and thus, had minimal changes in the roll angle of the camera. Apurba Das et al. [2] proposed a binarization calculation by altering the Min-Between-Max Thresholding (MBMT) approach, and subsequently, named as MMBMT. The identification consequences of the proposed MMBMT were viewed as conspicuous with the worldwide MBMT. Here, the ground truth is considered as the pattern casing, and subsequently, the negligible blunder can actuate uncommon change in execution, and in this way, the system requires manual discovery. Soonhong Jung et al. [3] proposed the path identification model dependent on spatiotemporal pictures. The technique executed tasks, like binarization and scaling based on the dominant parallel lines for the lane detection. The major advantage of the scheme was that it required only less computational time in comparison to other methods. The detection rate is very slightly improved. Ju Han Yoo et al. [4] developed the vigorous lane recognition scheme by means of the desertion point assessment model. The utilization of the vanishing point scheme improved the detection rate in different environments. The candidate line segments extracted for the detection process can differ from the ground value while executing the same in 3D space.

Xinxin Du, and Kok Kiong Tan [5] built up the viable stereo 3D reproduction technique for the path discovery, and the model recognized the path with the assistance of the recreation system. The plan was appropriate for the mechanized vehicle framework attributable to its high exactness. Likewise, the plan can be considered as the improvement to the vehicle path level restriction. The plan can think of some as street includes as the path markings, bringing about false location. Jianwei Niu et al. [6] presented a lane detection approach with Two-stage Feature Extraction (LDTFE). 
The LDTFE model has the ability to adjust difficult scenarios, like small line segments and line curvatures. In certain cases, the road may have nonexistent lines leading to lane detection failure. Heba Aly et al. [7] presented a lane detection model commonly, referred as LaneQuest. The LanQuest scheme helped to detect the road lanes using the smart phone, and also, the scheme was energy efficient. Utilizing improper cameras alongside the PDAs had expanded the likelihood of path location in outrageous climate conditions. Son Lam Phung [8] proposed the vision-based algorithm for aiding the pedestrian in the lane detection. The scheme had less complex structure, and thus, has high robustness and accuracy. The model failed to recognize the road lanes in the strong shadow conditions.

\section{CHALLENGES}

Different challenges prevailing in the lane discovery approach are clarified as follows:

- Lane detection approaches faces difficulties in dealing with the perspective distortion. In [10], the problem of the perspective distortion is eliminated by using the ridge feature and Inverse Perspective Mapping (IPM) based features. But, in the real case, further factors need to be considered[35].

- Most works adopt the IPM transformation for converting the image captured with the view picture from the bird's eye. In the transformed picture, the lanes are seen to be perpendicular, and detection is carried out using the simple filters or geometric constraints. The presence of obstacles in the image can drastically reduce the effectiveness of the algorithm [4].

- Using the Vp based approach [21] for lane detection has improved effectiveness as they consider the roads as the parallel planes. They reduce the false detection by filtering the lanes not passing through the $\mathrm{Vp}$. But, the correct detection of $\mathrm{Vp}$ is necessary for improving the effectiveness of the algorithm. Presence of noise and occlusion makes the Vp detection to be difficult [4].

- Generating the binary image from the original camera image can help in the lane detection approach. The techniques like as Otsu's, Neuro-visually inspired figure-ground segregation (NFGS), and adaptive binarization using integral image (ABII) generate the binary images for the detection, but the image cannot be further used for generating the ground truth information [2].

- Hough transform procedure be used for the detection of lanes. Using the hough transform for the detection can result in high false positive rate as the technique cannot clearly differentiate between the spurious lines and the shadows [4].

\section{METHODS USED AND FLOW OF PROPOSED SYSTEM}

This area describes the proposed multi-path location strategies and acknowledgment for driver help systems..

\section{A. Capturing Of The Lane Pictures From Road.}

Capturing of the lane pictures from road is most difficult task for the identification of lanes from the road many researchers uses the information obtained from the shape of the strip of lane.

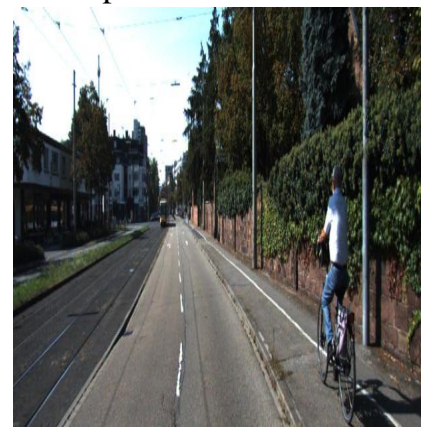

a)

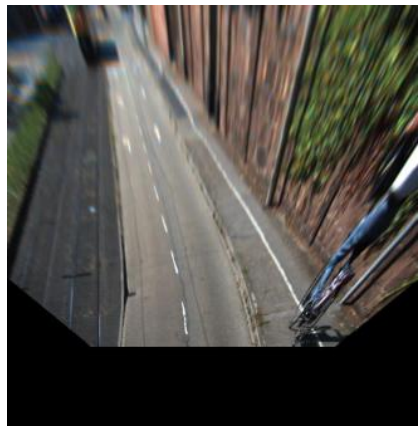

b)
Fig. 2. a) Input Camera Picture b) View Picture From The Bird's Eye.

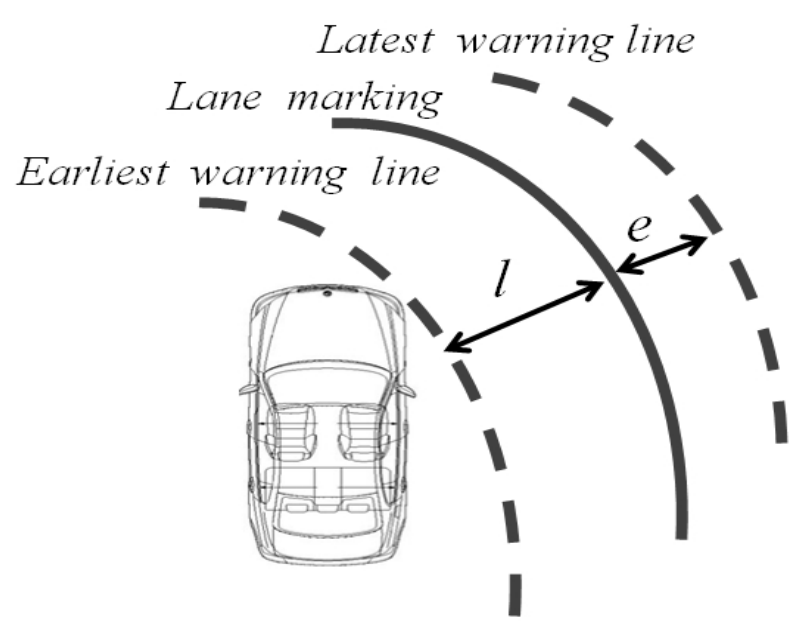

Fig. 3.Different Lines on the Road [36].

It is very difficult to capture an accurate picture of road for the identification of lane. For the capturing of the lane pictures from road in this article uses a good camera with 14 megapixel and with good sharpness and resolution. The pictures obtained from this camera has little number of noises. Also the camera gives more reliable pictures due to the infra red used in this. Also this camera gives good picture in different lighting conditions.

Moreover we acquire proper light of within street path design. Figure 2 shows the a) Input camera image b) view picture from the bird's eye. Figure 3 shows the different lines on the road. The transformation of an image is done in matlab which generate a top view perspective of an image as shown in figure 2 .

\section{B. Inverse Perspective Mapping}

The inverse perspective mapping scheme converts the numerous lane image to view picture from the bird's eye by applying various transformation schemes. The major aim of the image transformation process is to convert the multiple lane images as the images suitable for the camera frame[31]. The procedure for the conversion is depicted below:

Initially, the multiple road lane images have a lot of obstacles and it is avoided based on the body scale obstacle avoidance scheme. 


\section{(DCNN) Deep Convolution Neural Network Classifier and (EW-CSA) Earth Worm-Crow Search Algorithm for Lane Detection}

Consider the observer is moving in the horizontal plane with a translation constant $\mathrm{c}$, and the motion vector relative to a camera frame is represented as $\mathrm{dp} / \mathrm{dc}=-\mathrm{c}$.

Here, a constant motion field is considered and it is represented as $\mathrm{M}(\mathrm{x}, \mathrm{y}, \mathrm{z})$. The motion field projection is expressed by the following equation,

$$
\mathbf{M}^{\prime}(\varphi(\mathrm{p}))=\frac{\mathrm{d} \varphi(\mathrm{p})}{\mathrm{dt}}=-\mathrm{J} \varphi(\mathrm{p}) \cdot \mathrm{c}
$$

where, $\mathrm{J}$ refers to the Jacobian matrix considered for the perspective projection of the image. As the next step, the inverse perspective mapping is applied to the above expression, so as to find the transformed image, and it is expressed as,

$$
\mathrm{M}^{\mathrm{T}}=-\mathrm{J} \varphi(\mathrm{p}) \cdot \mathrm{c}=-\mathrm{J} \varphi^{\mathrm{T}}(\mathrm{p}) \cdot \mathrm{c}
$$

And the transformation of the multiple lane images $M$ to view picture from the bird's eye $\mathrm{M}^{\mathrm{T}}$ depends on the Inverse perspective mapping transformation parameter $\mathrm{T}$ and it is expressed as,

$$
M^{\prime T}=-T\left(\begin{array}{ccc}
-\frac{1}{\mathrm{p}^{3}} & 0 & -\frac{\mathrm{p}_{1}}{\frac{2}{3}} \\
0 & -\frac{1}{\mathrm{p}^{3}} & \frac{\mathrm{p}_{1}}{\mathrm{p}^{\frac{2}{3}}}
\end{array}\right)\left(\begin{array}{l}
\mathrm{c}_{1} \\
\mathrm{c}_{2} \\
\mathrm{c}_{3}
\end{array}\right)
$$

The ego motion of the image has been bound with the emotion plane and thus, the transformed image can be represented as,

$$
\mathrm{M}^{\mathrm{T}^{\mathrm{T}}}=\frac{\mathrm{T}}{\mathrm{p}_{3}}\left(\begin{array}{l}
\mathrm{c}_{1} \\
\mathrm{c}_{2}
\end{array}\right)
$$

Finally, view picture from the bird's eye transformed from the multiple lane image, is represented as,

$$
\left\|\mathbf{M}^{\prime T}\right\|=\left|\frac{\mathrm{T}}{\mathrm{T}-\text { elev }}\right|\|\mathrm{c}\|
$$

where, elev represents the elevation point and it has the value as $\mathrm{T}+\mathrm{p}_{3}$. Figure 4 shows the Bird's eye view by using Inverse perspective mapping transform.

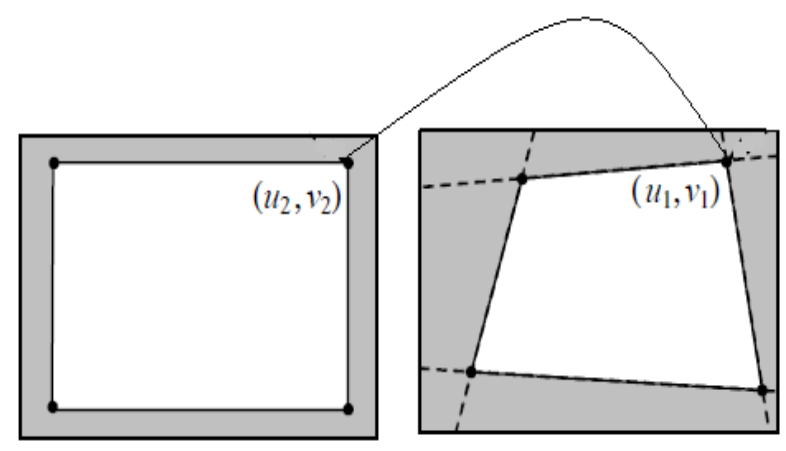

Fig. 4.Bird's Eye View by Using Inverse Perspective Mapping transform.

\section{Pre-processing and De-Noising}

To increase different features of the lane pictures, the pre-processing stage is used. The various features like as signal to noise ratio, intensity and contrast of picture are increases due to the processing stage. In this pre-processing stage the unwanted distortion is removed from the picture and if upgrades the picture which is useful for further processing.

The pre-processing stage only enhances the picture it does not increases the information presents in the picture. The standard range $[0,1]$ is utilized for the lane detection picture. This intensity range is most suitable for this system. Also to increase the intensity and the contrast of the picture captured from the road the diffusion filter is used [11]. The isotropic filter is used to increase the signal to noise ratio. The brightness transformation on the lane pictures is done on Matlab software. Also the median filter is used to remove the noise shot and noises due to the impulses. The weighted median filter (WM) is used to remove lane information and rank order information from the road lane picture.

\section{Transformation of Image using Hough Transform}

The Hough transform is used to remove the features of image lines, circles, ellipse and different shapes are detected by the Hough transform. The Hough transform which is used for the detection of line is generally called as accumulator. The equation for the detection of line for Hough transform is given by.

$$
\mathrm{x}=\mathrm{p} \cos \alpha+\mathrm{q} \sin \alpha
$$

Where, the quantized values $(x, \alpha)$ is calculate. The Hough transform is applied to the nearest pixels $(p, q)$ and from the information the line $(x, \alpha)$ is calculated. The values of quantized angle $\alpha$ and quantized distance $\mathrm{x}$ are represented in Matrix form in Hough transform.[17].

\section{E. DCNN Classifier.}

The transformed view picture from the bird's eye is applied to Deep Convolutional Neural Network (DCNN) classifier. The proposed model gets the numerous lane pictures this classifier changes the picture into its relating view picture from the bird's eye. The proposed approach is to clearly identify the view picture from the bird's eye for the participation image example ( multi-lane images).

\section{F. Lane Detection Through The Proposed EW-CSA Based DCNN Classifier}

DCNN classifier uses the birds eye picture view image as an input. This birds eye picture view image is used by DCNN classifier to differentiate the path lane. To prepare this DNNN classifier, a combination of (EWA) and (CSA) algorithms are used. [19].

$$
M_{k, l}=\frac{\eta_{k} * H^{k, m}-1}{\eta_{k} * H^{k, m}}\left(K_{l} * L-\frac{\eta_{k} * H^{k, m} * h^{l, m}}{1-\eta_{k} * H^{k, m}}\right)
$$


where, $\prod \mathrm{k}$ shows the range of arbitrary number and it is 0 to 1 . The flight length is $\mathrm{Hkm}$, The weight vector is represented as $\mathrm{k} 1$. The distribution of Cauchy random number is represented by $\mathrm{L}$. The best arrangement of memories for mth iteration is shown by hlm.

The DCNN contains the organized staged arrangement of the CNN layers, and the layers in the DCNN [4] are complication layer, likewise referred as (conv) layers, pooling (POOL) layers, and Fully Connected (FC) layers. On behalf of the preparation, the DCNN acquires the 10,000 foot view picture, and it is straightforwardly given to the Convolutional layer[9].

The POOL layer plays out the undertaking of subsampling, and the output is gotten from the FC layer. The layers of the proposed EW-CSA based DCNN is clarified below:

\section{G. Convolutional Layers}

The convolutional layer obtains the bird's eye image and it is fed to the first Convolutional layer. The DCNN has several Convolutional layers interconnected by weight parameters. The bird's eye image and the weight are combined to figure a characteristic map for the training. Information from aConvolutional layer to additional is passed during the commencement function. a Convolutional layer of the DCNN is represented by the next expression,

$$
\mathrm{K}=\left\{\mathrm{K}_{1}, \mathrm{~K}_{2}, \ldots, \mathrm{K}_{\mathrm{v}}, \ldots, \mathrm{K}_{\mathrm{m}}\right\}
$$

where, $\mathrm{m}$ refer to total Convolutional layer count. The term $\mathrm{K}_{\mathrm{v}}$ shows the vth convolutional level in the Deep CNN. A production of the Convolutional level be calculated as follows,

$$
\left(Y_{d}^{v}\right)_{r, s}=\left(B_{d}^{v}\right)_{r, s}+\sum_{e=1}^{J_{1}^{e-1}} \sum_{p=-n_{1}^{j}}^{n_{1}^{j}} \sum_{q=-n_{2}^{j}}^{n_{2}^{j}}\left(G_{d, e}^{v}\right)_{p, q} *\left(Y_{e}^{v-1}\right)_{r+p, s+q}
$$

where, * refer to the conv operator,

$\left(Y_{e}^{v-1}\right)_{r+p, s+q}$ indicate the feature map input to the conv layer,

$J_{1}^{e-1}$ refer to the features from previous Convolutional layer,

$\left(B_{d}^{v}\right)_{r, s}$ denote the bias and

$\left(G_{d, e}^{v}\right)_{p, q}$ represents a weight of a convolution layer.

\section{H. Pooling (POOL) Layer and Rectified Linear Unit (ReLU)}

ReLU gives the actuation capacity to controlling the Convolutional layer yield, and it guarantees the viability. The yield of the ReLU is spoken to as,

$$
Y_{d}^{v}=f u n\left(Y_{d}^{v-1}\right)
$$

For the vth layer, the activation function is given by fun (). The pool layer takes the ReLU output. Any weights and bias does not processed by the pool layer.

Fully Connected layers: The information getting from the pooling layer is given to the fully connected layers. The information obtained from these fully connected layers is utilized for the formula.

$$
\mathrm{Z}_{\mathrm{d}}^{\mathrm{v}}=\mathrm{T}\left(\mathrm{b}_{\mathrm{d}}^{\mathrm{v}}\right) \text { with } b_{d}^{j}=\sum_{e=1}^{J_{1}^{e-1}} \sum_{r=1}^{J_{2}^{e-1}} \sum_{s=1}^{J_{3}^{e-1}}\left(U_{d, e, r, s}^{v}\right) \cdot\left(Y_{d}^{v-1}\right)_{r, s}
$$

where, The weight interconnection $(r, s)$ indicated by $\left(U_{d, e, r, s}^{v}\right)$ for the feature map of eth feature of $\mathrm{v}-1$ layer and $\mathrm{J}$ layer of dth unit. The weights obtained from these 3 layers plays an important role for the completion of output, and therefore it is considered as an optimization algorithm. D $€$ $\{B, G, U\}$ represents optimization algorithm, which is used to find weights of deep CNN.

\section{Algorithmic Description Of The Proposed EW-CSA}

1) Initialization: EWA algorithm initializes earthworm population, and regulates the optimization procedure through the reproduction behavior. The earthworm initialized by the proposed EW-CSA is expressed as,

$$
\mathrm{X}=\left\{\mathrm{X}_{\mathrm{ij}}\right\}
$$

where, Xij specifies the earthworm in the solution space and the value of $i$ and $j$ extends in range of 1 to $D$.

2) Fitness criterion: After randomly initializing the earthworm population, the position of the earthworm is sorted out based on its position. Here, the earthworm having the best position is said to have the best solution.

3) Position update: The reproduction behavior of the earthworm is carried out by considering various crossover and mutation operations. Here, single point crossover, multipoint cross over and uniform cross over are considered for updating the positions of the earthworms. The EWA considers the reproduction and the regeneration activities of the earthworm for carrying out the optimization task. Figure 5 shows the crow search algorithm[10]. EWA constantly increases the population size, as the earthworm having the best fitness is retained and directly passed on to the next iteration. The existing EWA algorithm faces issues related to the local minima trap and it can be avoided by performing the update based on the Cauchy mutation and it is represented as,

$$
\mathrm{X}_{\mathrm{ij}}{ }^{\prime}=\mathrm{X}_{\mathrm{ij}}+\mathrm{Q}_{\mathrm{j}} * \mathrm{Z}
$$

where, Qj refers to the weight vector, and $\mathrm{Z}$ represents the Cauchy distribution random number. Here, the Cauchy mutation based update is modified based on CSA [25], and the position update expression of the CSA is expressed as,

$$
X^{i, i t e r+1}=X^{i, i t e r}+\mu_{i} * *\left(w^{j, i e r}-X^{i, i t e r}\right)
$$




\section{(DCNN) Deep Convolution Neural Network Classifier and (EW-CSA) Earth Worm-Crow Search Algorithm for Lane Detection}

where, $L^{i, \text { iter }}$ refers to the flight length, and $w^{j, i e r}$ indicates the memory of the best solution. The term $\mu_{i}$ indicates the random number in the range $[0,1]$. Now, modifying the above equation,

$$
\mathrm{X}^{\mathrm{i}, \text { iter }}=\frac{1}{1-\mu_{\mathrm{i}} * \mathrm{~L}^{\mathrm{i}, \text { iter }}}\left(\mathrm{X}^{\mathrm{i}, \text { iter }+1}-\mu_{\mathrm{i}} * \mathrm{~L}^{\mathrm{i}, \text { iter }} * \mathrm{~W}^{\mathrm{j}, \text { ier }}\right)
$$

Now, substituting the equation (13) in equation (11) to regulate the optimization process, and the modified expression is given as,

$$
\mathrm{X}_{\mathrm{ij}}{ }^{\prime}=\frac{1}{1-\mu_{\mathrm{i}} * \mathrm{~L}^{\mathrm{i}, \text { iter }}}\left(\mathrm{X}_{\mathrm{ij}}{ }^{\prime}-\mu_{\mathrm{i}} * \mathrm{~L}^{\mathrm{i}, \text { iter }} * \mathrm{w}^{\mathrm{j}, \text { ier }}\right)+\mathrm{Q}_{\mathrm{j}} * \mathrm{Z}
$$

Now, modifying the above equation,

$$
\mathrm{X}_{\mathrm{ij}}{ }^{\prime}=\frac{\mu_{\mathrm{i}} * \mathrm{~L}^{\mathrm{i}, \text { iter }}-1}{\mu_{\mathrm{i}} * \mathrm{~L}^{\mathrm{i}, \text { iter }}}\left(\mathrm{Q}_{\mathrm{j}} * \mathrm{Z}-\frac{\mu_{\mathrm{i}} * \mathrm{~L}^{\mathrm{i}, \text { iter }} * \mathrm{~W}^{\mathrm{j}, \text { ier }}}{1-\mu_{\mathrm{i}} * \mathrm{~L}^{\mathrm{i}, \text { iter }}}\right)
$$

The above expression refers to the modified Cauchy mutation expression for the proposed EW-CSA algorithm, and based on this expression, the population update is carried on.

4) Termination: The expression (15) is used for the position update, and on each iteration, the weights are regulated through the fitness. After a certain iteration limit, the optimal weights are passed through the proposed EW-CSA based DCNN classifier for the lane recognition.

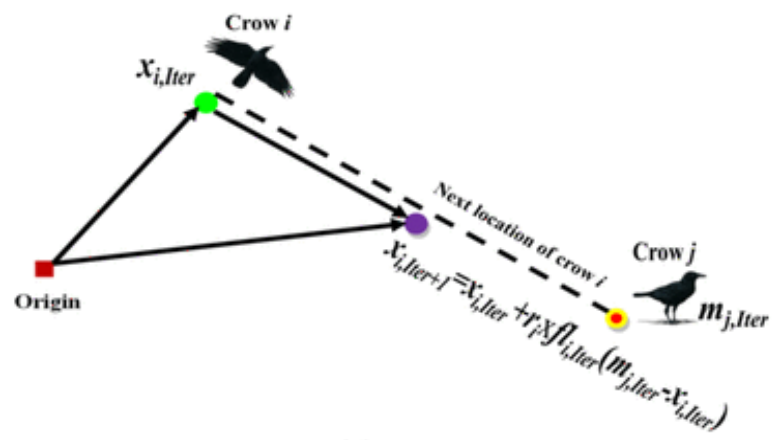

(a) $f \leq 1$

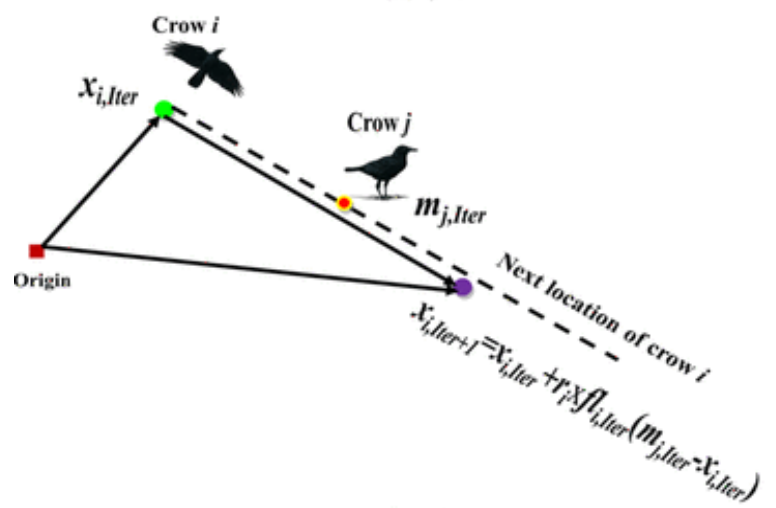

(b) $f l>1$
Fig. 5. Crow Search Calculation a) To fl $<1$ b)

To fl > 1 [35].

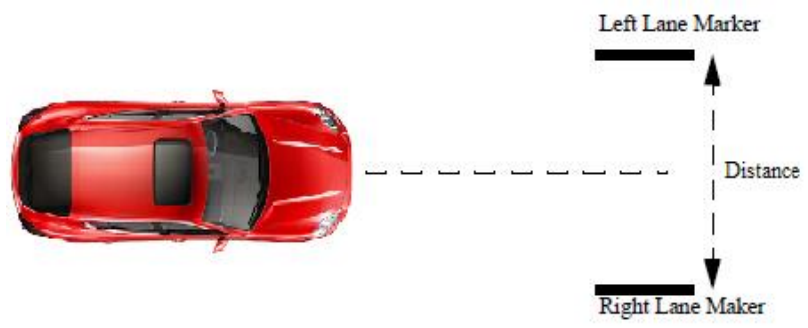

Fig. 6.Distance Calculation using Bhattacharya Distance Separation Method.

The optimal weights for the DCNN are tuned based on the proposed EW-CSA algorithm, and the lane detection is carried out.

\section{J. Bhattacharya Distance Separation}

The Bhattacharya distance separation [1] is utilized to find the distance between the targets and the grid points. The similarity of 2 probability distributions are utilized to find the distance separation. There are ' $n$ ' number of centroids are used in this article to identify the distance and from these ' $n$ ' number of centroids the Bhattacharya distance selects the grid. The selected grids for Bhattacharya distance are matches with points and minimum distance target is selected. The example diagram of Bhattacharya distance separation is shown in diagram 6. and the Bhattacharya distance is calculated by using formula

$B_{D}(E, T)=\frac{1}{4} \ln \left(\frac{1}{4}\left(\frac{\beta^{2}{ }_{E}}{\beta^{2}{ }_{T}}+\frac{\beta^{2}{ }_{T}}{\beta^{2}{ }_{E}}+2\right)\right)+\frac{1}{4}\left(\frac{\left(\tau_{E}-\tau_{T}\right)^{2}}{\beta^{2}{ }_{E}+\beta^{2}{ }_{T}}\right)+\frac{1}{4}\left(\frac{r_{E}-r_{T}}{\beta^{2}{ }_{E}+\beta^{2}{ }_{T}}\right)$

where, the in between distance from grid and the target is given by $\mathrm{B}_{\mathrm{D}}(\mathrm{E}, \mathrm{T})$,

$\beta_{E}^{2}$ be $\mathrm{E}^{\text {th }}$ distribution variance,

$\beta^{2}{ }^{2}$ be $\mathrm{T}^{\text {th }}$ distribution the variance,

$\tau_{E}$ be $\mathrm{E}^{\text {th }}$ distribution of the mean , $\tau_{T}$ be $\mathrm{T}^{\text {th }}$ distribution of the mean, and the kurtosis of the centroid are rE and rT. The space designed by Bhattacharya distance division technique is belongs to exacting object.

\section{K. Segmentation of Picture}

The segmentation of picture is presents in the detection module in this work. In this detection module the segmentation is done on the pictures obtained from the road lane. From this picture in segmentation capture the exact area, pattern of lane and distance between the lane. The unwanted background pictures presents in a road lane images are also removed by this segmentation division method. [The first step of this proposed lane detection system is segmentation system. The detection of lane border line, layout, centers etc. is the main purpose of this process. 


\section{Region Based Iterative Seed Method For Multiple Lane Identification}

This Region based Iterative seed Method for Multiple lane identification method is used for the identification of multiple lanes. The sparking method is utilized for this purpose. When the segmentation of an image is completed the samples of input image are processed through sparking method and after the sparking process is completed the input images is transferred to region based iterative seed method to identify the lanes. In this algorithm different matrices are obtained by partitioning the picture and randomly any one target is selected. When this process is completed the distance between the target and grid is calculated by using Bhattacharya distance calculation.[29]. Figure 7 shows the different challenges occurs while road lane identification.
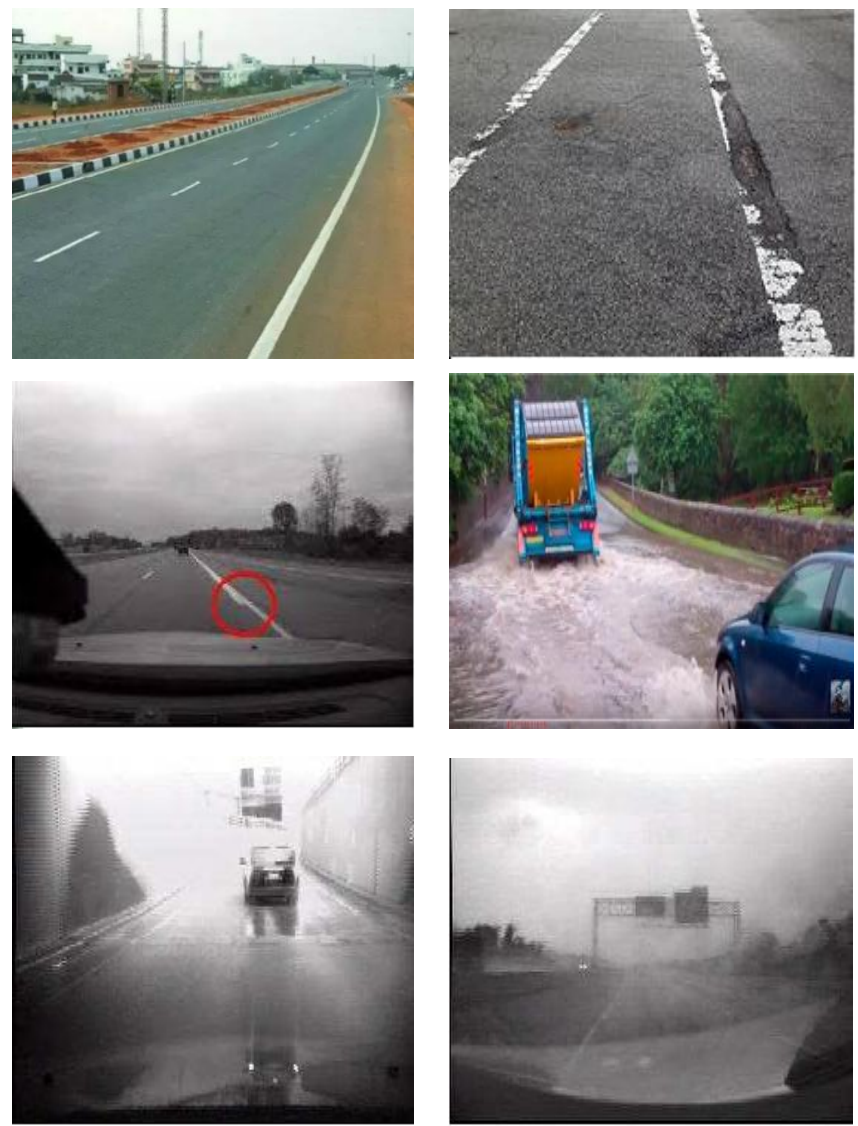

Fig. 7.Different Challenges for Lane Detection [37].

\section{BLOCK DIAGRAM OF PLANNED METHODOLOGY}

In this section the block diagram description of proposed multi-lane detection system. The multi-lane image obtained from the camera is feed to the pre-processing and de-noising block to remove a noise presents in the image. It is also used to improve the different parameters a path identification pictures, for instance, differentiate, force, sign to clamor proportion, and so forth.

The output image obtained from the pre-processing and de-noising stage is given to a transformation block. The transform using Hough is used for the image transformation. The transform using Hough detects the edges from a Projected input image and view picture from the bird's eye.

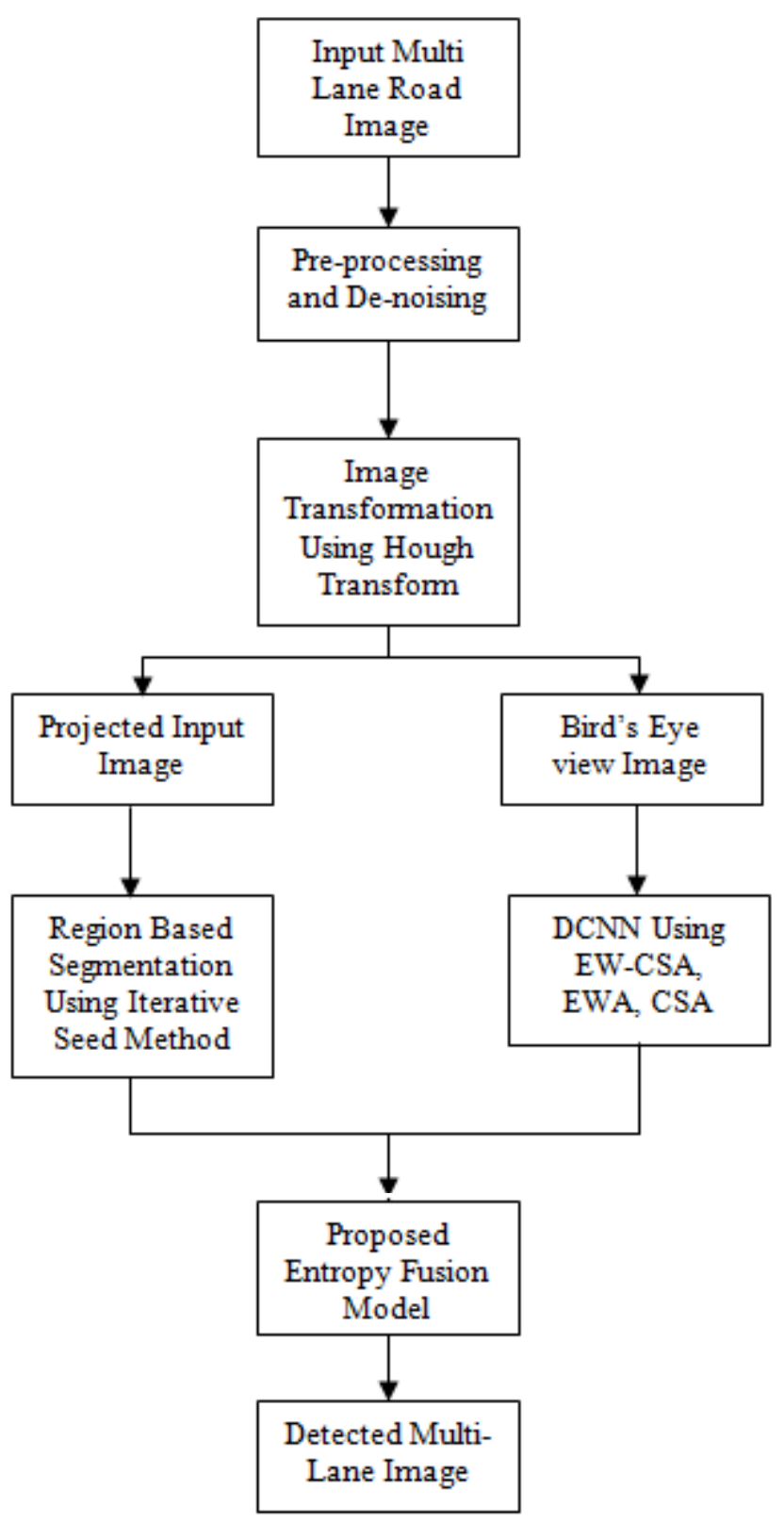

Fig. 8.Block Diagram Of Multi-Lane Detection.

The projected input image is fed to the segmentation using iterative seed method which is utilized to detect a lane. The view picture from the bird's eye is fed to Deep Convolutional Neural Network (DCNN) classifier.

It is the fusion of Earthworm optimization algorithm (EWA) and Crow Search Algorithm (CSA), is used for choosing the supreme weights via preparing the DCNN. The images obtained from the iterative seed method and DCNN classifier is fed to the Proposed Entropy Fusion representation to fuse a separated images in transformation step. Figure 8 represents the block figure of planned multi-lane recognition structure.

\section{EXPERIMENTAL RESULTS}

In this segment, the stepwise experimental results are explained. Figure 9 shows the captured picture of road with lane, in this image the road lanes are clearly seen. Figure 10 shows the Bird's eye picture of road with lane after transformation.

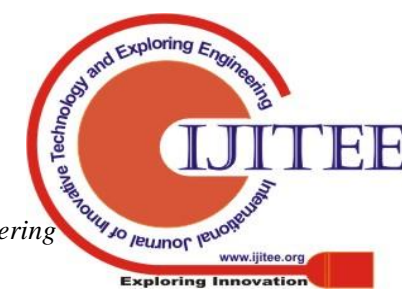


(DCNN) Deep Convolution Neural Network Classifier and (EW-CSA) Earth Worm-Crow Search Algorithm for Lane Detection

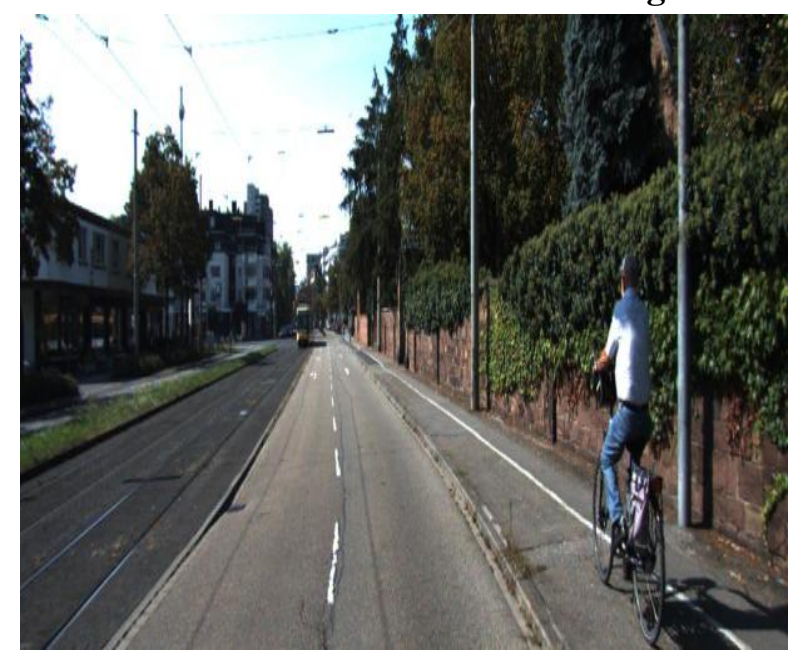

Fig. 9.Captured Picture of Road with Lane.

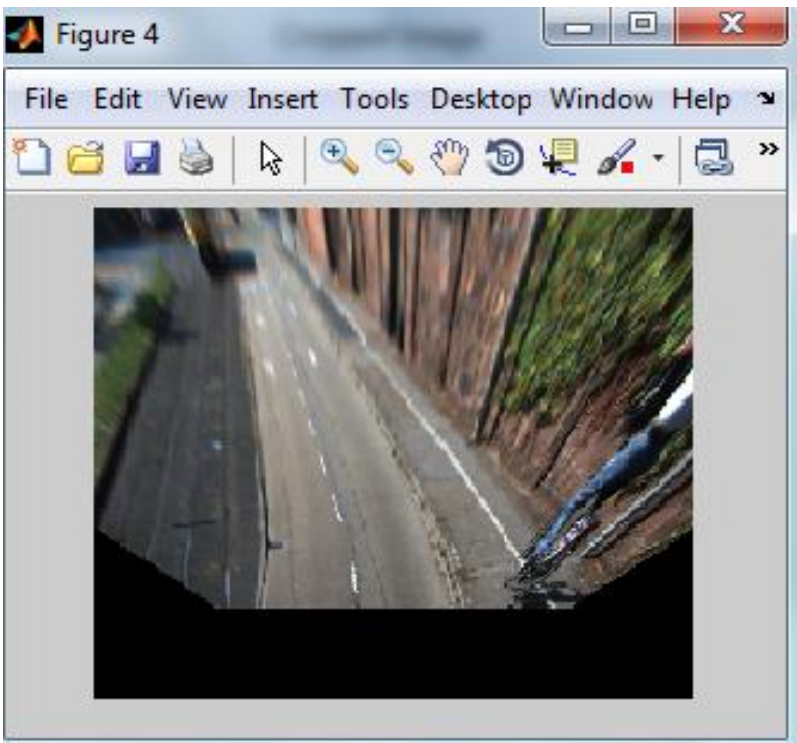

Fig. 10. Bird's Eye Picture of Road with Lane.

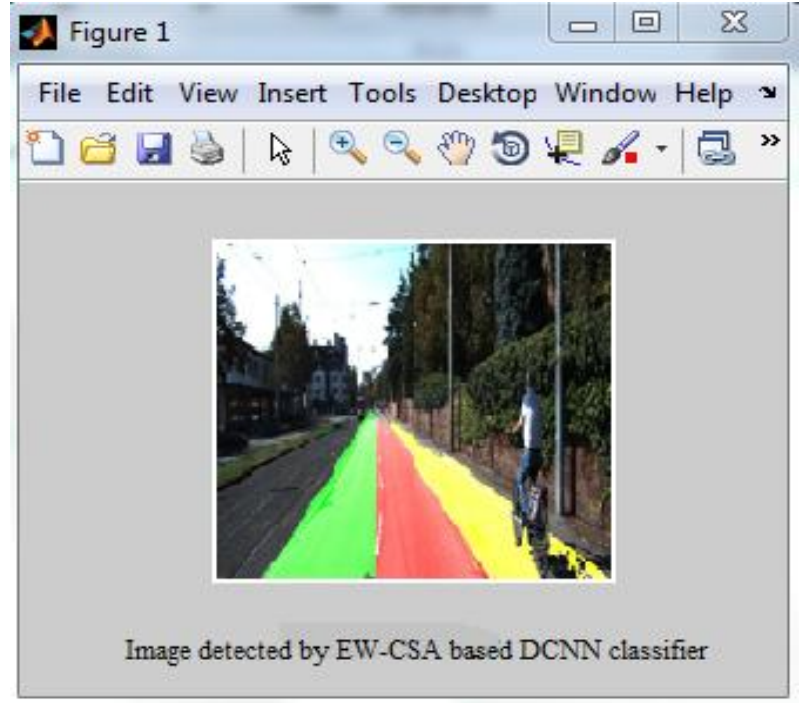

Fig. 11. Image Detected By EW-CSA Based DCNN Classifier.

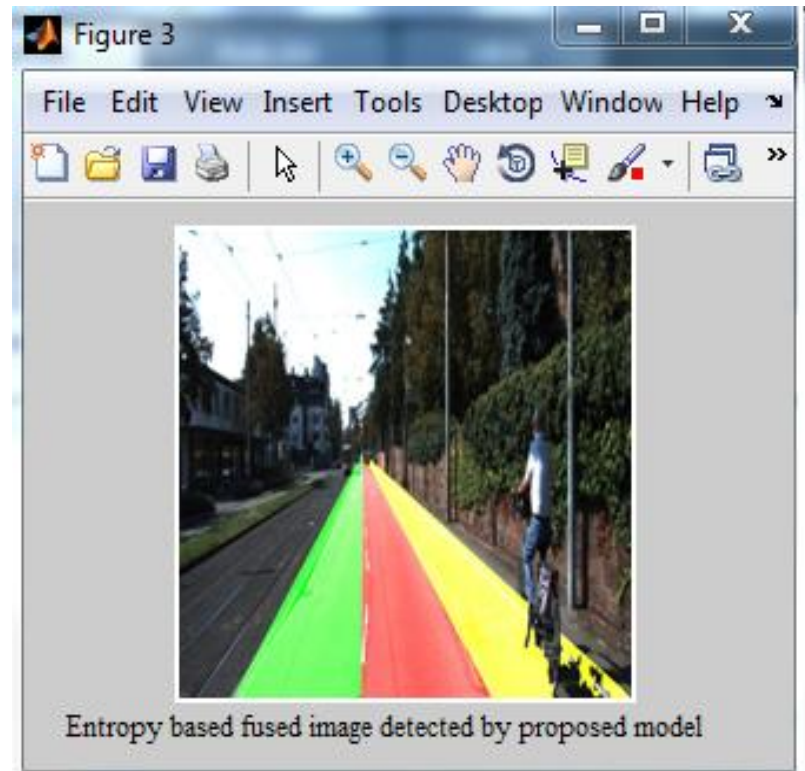

Fig. 12. Entropy Based Combined Representation Identified By Projected Model.

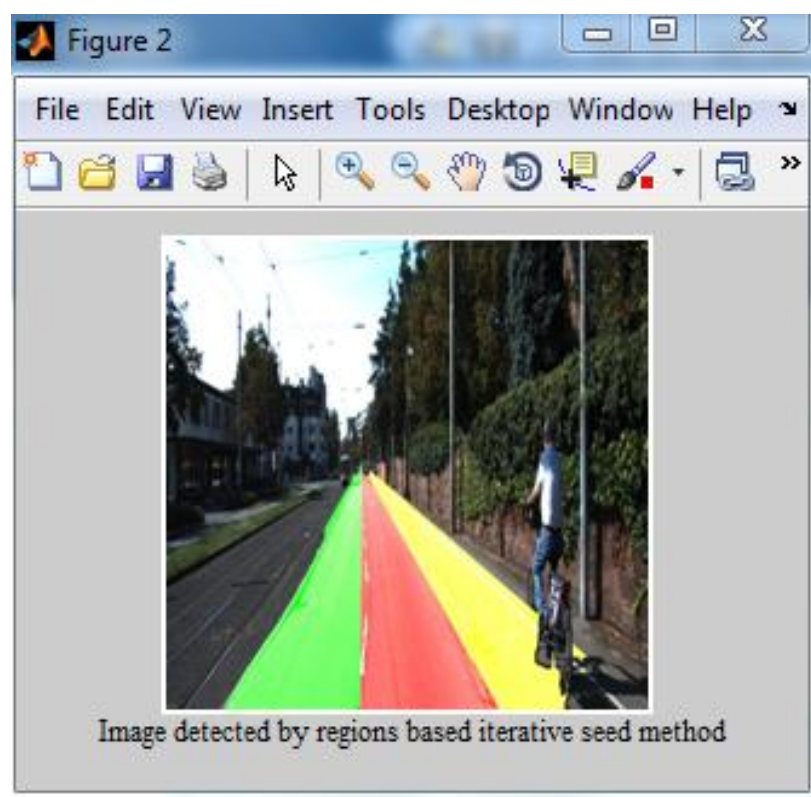

Fig. 13. Picture Identified By Regions Based Iterative Seed Algorithm.

In figure 11 the picture identified by EW-CSA based DCNN classifier which detects the lane, the colors Green, Red and Yellow are used to give the lane color.

In figure 12 entropy based combined representation identified by projected model be present.

Picture identified by regions based iterative seed method is shown in figure 13.

Table 2 demonstrates the relative discourse of various techniques as far as discovery precision, affectability and particularity.

From this table plainly the precision of the techniques utilized is more than the other parameter. 
Table I Comparative discussion

\begin{tabular}{|c|c|c|c|}
\hline Methods & $\begin{array}{c}\text { Detection } \\
\text { Accuracy }\end{array}$ & Sensitivity & Specificity \\
\hline DVPE & 0.9141 & 0.8712 & 0.74 \\
\hline $\begin{array}{c}\text { EW-CSA based } \\
\text { DCNN }\end{array}$ & 0.9785 & 0.9788 & 0.7655 \\
\hline DCNN & 0.9838 & 0.9762 & 0.7041 \\
\hline MMBM & 0.9107 & 0.9417 & 0.7440 \\
\hline $\begin{array}{c}\text { Region based } \\
\text { Iterative Seed }\end{array}$ & 0.9889 & 0.9916 & 0.8863 \\
\hline
\end{tabular}

\section{CONCLUSION}

In this manuscript, a desired approach for lane exposure be presents. The multiple lane pictures obtained by a camera are fed as the training input to the proposed model, and they are subjected to the image inverse perspective mapping transformation. After the transformation, the view picture from the bird's eye is provided to a DCNN classifier for the lane detection. The optimal weights for the DCNN are tuned based on the proposed EW-CSA algorithm, and the lane detection is carried out. The proposed EW-CSA algorithm is developed in this paper so as to select the optimal weights for the DCNN training. The performance is evaluated based on detection accuracy, sensitivity, and specificity. The proposed EW-CSA based DCNN classifier achieved improved results with the values of $0.99512,0.9925$, and 0.995 for the detection accuracy, sensitivity, and specificity. The test results demonstrate that the picture discovery strategy is precise. The proposed work is planned in MATLAB, picture handling tool compartment for perusing the photos and play out the various tasks like as transformation, pre-processing, DCNN, EW-CSA, etc..

\section{REFERENCES}

1. Umar Ozgunalp, Rui Fan, Xiao Ai, and Naim Dahnoun, "Multiple Lane Detection Algorithm Based on Novel Dense Vanishing Point Estimation", IEEE Transactions On Intelligent Transportation Systems, Vol. 18, No. 3, March 2017.

2. Apurba Das, Member, Siva Srinivasa Murthy, and Upendra Suddamalla, "Enhanced Algorithm of Automated Ground Truth Generation and Validation for Lane Detection System by M2BMT", IEEE Transactions On Intelligent Transportation Systems, Vol. 18, No. 4, April 2017.

3. Soonhong Jung, Junsic Youn, and Sanghoon Sull, "Efficient Lane Detection Based on Spatiotemporal Images", IEEE Transactions On Intelligent Transportation Systems, January 2015.

4. Ju Han Yoo, Seong-Whan Lee, Sung-Kee Park, and Dong Hwan Kim, "A Robust Lane Detection Method Based on Vanishing Point Estimation Using the Relevance of Line Segments", IEEE Transactions On Intelligent Transportation Systems", vol.18, no.12, February 2017.

5. Xinxin Du, and Kok Kiong Tan, "Comprehensive and Practical Vision System for Self-Driving Vehicle Lane-Level Localization", IEEE Transactions On Image Processing, Vol. 25, No. 5, May2016.

6. Jianwei Niu, JieLu, Mingliang Xu, PeiLv, and Xiaoke Zhao, "Robust Lane Detection using Two-stage Feature Extraction with Curve Fitting", Pattern Recognition, vol 59, pp.225-233, November 2016.

7. Heba Aly, Anas Basalamah, and Moustafa Youssef, "Robust and ubiquitous smartphone-based lane detection", Pervasive and Mobile Computing, 2015.

8. Son Lam Phung, Manh Cuong Le , and Abdesselam Bouzerdoum, "Pedestrian lane detection in unstructured scenes for assistive navigation", Computer Vision and Image Understanding, vol.149, pp.186-196, 2016.

9. Rasmussen, C., Korah, T.: On-vehicle and aerial texture analysis for vision-based desert road following. In: CVPR workshop on machine vision for intelligent vehicles. (2005) III: $66\{66$

10. Montemerlo, M., et al.: Junior: The Stanford entry in the Urban Challenge. Journal of Field Robotics 25(8) (2008) 569\{597 25

11. Gao, T., Aghajan, H.: Self lane assignment using egocen- tric smart mobile camera for intelligent GPS navigation. In: Workshop on Egocentric Vision. (2009) 57\{62

12. Jiang, Y., Gao, F., Xu, G.: Computer vision-based multiple- lane detection on straight road and in a curve. In: ImageAnalysis and Signal Processing. (2010) $114\{117$

13. Wu, S., Chiang, H., Perng, J., Chen, C.,Wu, B., Lee, T.: The heterogeneous systems integration design and implementation for lane keeping on a vehicle. IEEE Transactions on Intelligent Transportation Systems 9 (2008) 246\{263

14. Hofmann, U., Rieder, A., Dickmanns, E.: Radar and vision data fusion for hybrid adaptive cruise control on highways. Machine Vision and Applications 14 (2003) 1, 42\{49

15. Batavia, P.H.: Driver-adaptive lane departure warning sytems. CMU-RI-TR-99-25 (1999)

16. Meng-Che Chuang, Jenq-Neng Hwang, and Kresimir Williams", A Feature Learning and Object Recognition framework for underwater fish images", IEEE transactions on image processing, vol.25, no.4, pp.1862-1872, 2016.

17. Yuichi Saito, Makoto Itoh, and Toshiyuki Inagaki,"Driver Assistance System with a Dual Control Scheme: Effectiveness of Identifying Driver Drowsiness and Preventing Lane Departure Accidents",IEEE transactions on human-machine systems, March 2016.

18. K. Bengler, K. Dietmayer, B. Farber, M. Maurer, C. Stiller, and H Winner, "Three decades of driver assistance systems: Review and future perspectives," IEEE Intelligent Transportation Systems Magazine, vol. 6, no. 4, pp. 6-22, April 2014

19. Ju Han Yoo, Seong-Whan Lee, Sung-Kee Park, and Dong Hwan Kim, "A Robust Lane Detection Method Based on Vanishing Point Estimation Using the Relevance of Line Segments", IEEE Transactions On Intelligent Transportation Systems", vol.18, no.12, February 2017.

20. Gurveen Kaur, and Dinesh Kumar,"Lane Detection Techniques: A Review", International Journal of Computer Applications, vol.112, no 10, pp.0975 - 8887, February 2015.

21. Seokju Lee, Junsik Kim, Jae Shin Yoon, Seunghak Shin,OleksandrBailo, Namil Kim Tae-Hee Lee, Hyun Seok Hong, Seung-Hoon Han, and In So Kweon, "VPGNet: Vanishing Point Guided Network for Lane and Road MarkingDetection and Recognition", In Proceedings of the IEEE International Conference on Computer Vision, pp. 1947-1955, 2017.

22. Alexander Rakhlin, Alexey Shvets, Vladimir Iglovikov, and Alexandr A. Kalinin, "Deep Convolutional Neural Networks for Breast Cancer Histology Image Analysis", In proceedings of International Conference on Image Analysis and Recognition ICIAR, Image Analysis and Recognition, pp. 737-744, 2018.

23. Jun Li, XueMei, Danil Prokhorov, and Dacheng Tao,"Deep Neural Network for Structural Predictionand Lane Detection in Traffic Scene",IEEE transactions on neural networks and learning systems, January 2016.

24. Mane, V.M. and Jadhav, D.V., "Holoentropy enabled-decision tree for automatic classification of diabetic retinopathy using retinal fundus images," Biomedical Engineering/Biomedizinische Technik, vol.62, no.3, pp.321-332, 2017.

25. The KITTI Vision Benchmark Suite taken from "http://www.cvlibs.net/datasets/kitti/eval_road.php", accessed on August 2018.

26. Umar Ozgunalp, Rui Fan, Xiao Ai, and NaimDahnoun, "Multiple Lane Detection Algorithm Based on Novel Dense Vanishing Point Estimation", IEEE Transactions On Intelligent Transportation Systems, Vol. 18, No. 3, March 2017.

27. M. A. Sotelo, F. J. Rodriguez, L. Magdalena, L. M. Bergasa, and L. Boquete, "A color vision-based lane tracking system for autonomous driving on unmarked roads," Automotive Robotics , vol. 16, no. 1, pp. 95-116, January 2004.

28. López, J. Serrat, C. Cañero, F. Lumbreras, and T. Graf, "Robust lane markings detection and road geometry computation," International Journal of Automotive Technology, vol. 11, no. 3, pp. 395-407, 2010.

29. D. Schreiber, B. Alefs, and M. Clabian, "Single camera lane detection and tracking," in Proceedings on IEEE Transactions on Intelligent Transportation Systems, 2005, pp. 302-307.

30. Y. Wang, N. Dahnoun, and A. Achim, "A novel system for robust lane detection and tracking," Signal Processing, vol. 92, no. 2, pp. 319-334, 2012 . 
(DCNN) Deep Convolution Neural Network Classifier and (EW-CSA) Earth Worm-Crow Search Algorithm for Lane Detection

31. Y. Wang, E. K. Teoh, and D. Shen, "Lane detection and tracking using B-snake," Image and Vision Computing., vol. 22, no. 4, pp. 269-280, 2004.

32. Y. Wang, L. Bai, and M. Fairhurst, "Robust road modeling and tracking using condensation," IEEE Transactions on Intelligent Transportation Systems, vol. 9, no.4, pp. 570-579, Dec. 2008.

33. C. Rasmussen, "Grouping dominant orientations for ill-structured road following," in Proceedings on IEEE CVPR, 2004, vol. 1, pp. I-470-I-477.

34. https://www.google.com/search?tbm=isch\&sxsrf=ACYBGNSBkOTQL JChugxKa7CA0kul6erzXg\%3A1570211788010\&sa=1\&ei=zIeXXcQk wtrPuw_q8LDgCQ\&q=road+lane+detection+system\&oq=road+lane+d etection+system\&gs_l=img.3...144860.151727..152637...0.0..0.236.31 82.0j14j3 ..........1..gws-wiz-img.......0i8i30.YDdoTXHMPAE\&ved=0 ahUKEwjEuuLeloPlAhVC7XMBHWo4DJwQ4dUDCAY\&uact=5\#im grc=z7Sk3PYmPaOh7M:

35. https://www.google.com/search?tbm=isch\&sxsrf=ACYBGNSMq07H XnjeFV49L8uCIcEftEy91g\%3A1570211951491\&sa=1\&ei=b4iXXZb

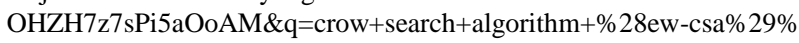
2C\&oq=cro\&gs_l=img.3.0.35i39j0i6719.251018.251574..253792...0.0 ..0.742.1139.0j1j1j6-1.....0....1..gws-wiz-img......0.xafvuLc0QmM\#im grc $=$ XvRC-FwhTbkMlM:

36. https://www.google.com/search?tbm=isch\&sxsrf=ACYBGNTBKB6Ky vqBAULkIfkAJX8toGtI_A\%3A1570212211199\&sa=1\&ei=c4mXXd_ mC7Xfz7sP27KUqA0\&q=lastest+warning+lane\&oq=lastest+warning +lane\&gs_l=img.3...112836.120562..120676...0.0..0.421.3890.0j8j9j0 j1 ..........1..gws-wiz-img.......35i39j0j0i67j0i10j0i10i24.sw8P29hFHO 0\&ved=0ahUKEwif7MeomIPlAhW173MBHVsZBdUQ4dUDCAY\&u act $=5 \#$ imgrc $=\mathrm{a} 2 \mathrm{~b} 1 \mathrm{aN} 3 \mathrm{eEDJS} 4 \mathrm{M}$ :

37. Recent Progress in Road and Lane Detection - A survey by Aharon Bar Hillel $\varnothing$ Ronen Lerner $\varnothing$ Dan Levi $\varnothing$ Guy Raz

\section{AUTHORS PROFILE}

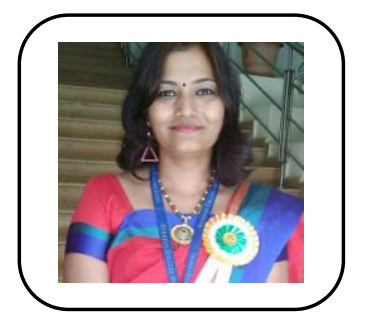

Suvarna Shirke-Pansambal is the Research Scholar at Bharath Institute of Higher Education and Research, Department of Computer Science \& Engineering, Chennai. She is working as an Assistant Professor in Atharva College of Engineering, Mumbai.

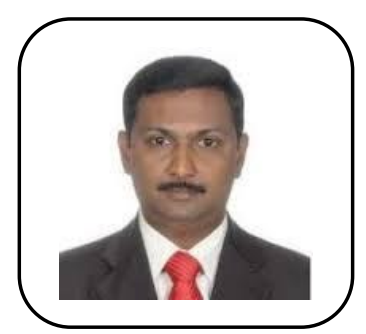

Dr.R.Udayakumar is Professor at Department of Information Technology, Bharath Institute of Higher Education and Research, Chennai, India. He has published more than 600 papers and he is Supervisor for Research Scholars at BIHER. 